\title{
Synergistic anticancer effect of acteoside and temozolomide-based glioblastoma chemotherapy
}

\author{
TAE WOONG HWANG ${ }^{1,2}$, DONG HUN KIM ${ }^{1,2}$, DA BI KIM ${ }^{1,2}$, TAE WON JANG ${ }^{3}$, GUN-HWA KIM ${ }^{4}$, \\ MINHO MOON ${ }^{5}$, KYUNG AH YOON ${ }^{6}$, DAE EUN CHOI ${ }^{2}$, JAE HO PARK ${ }^{7}$ and JWA-JIN KIM ${ }^{1,2,8}$
}

\author{
Departments of ${ }^{1}$ Medical Science and ${ }^{2}$ Nephrology, School of Medicine, Chungnam National University, Daejeon 35015; \\ ${ }^{3}$ Department of Medicinal Plant Resources, Andong National University, Andong, Gyeongsangbuk 36729; \\ ${ }^{4}$ Drug and Disease Target Team, Division of Bioconvergence Analysis, Korea Basic Science Institute, Cheongju, \\ Chungcheong 28119; ${ }^{5}$ Department of Biochemistry, College of Medicine, Konyang University, Daejeon 35365; \\ ${ }^{6}$ Department of Clinical Laboratory Science, Daejeon Health Sciences College, Daejeon 34504; \\ Departments of ${ }^{7}$ Pharmaceutical Science and ${ }^{8}$ Biomedical Science, Jungwon University, \\ Geosan, Chungbuk 28024, Republic of Korea
}

Received July 9, 2018; Accepted January 9, 2019

DOI: $10.3892 /$ ijmm.2019.4061

\begin{abstract}
Temozolomide (TMZ) is an alkylating agent commonly used as a first-line treatment for high-grade glioblastoma. However, TMZ has short half-life and frequently induces tumor resistance, which can limit its therapeutic efficiency. In the present study, it was hypothesized that combined treatment with TMZ and acteoside has synergistic effects in glioblastoma therapy. Using cell viability and wound-healing assays, it was determined that this treatment regimen reduced cell viability and migration to a greater extent than either TMZ or acteoside alone. Following previous reports that TMZ affected autophagy in glioma cells, the present study examined the effects of TMZ + acteoside combination treatment on apoptosis and autophagy. The TMZ + acteoside combination treatment increased the cleavage of caspase- 3 and levels of B-cell lymphoma 2 (Bcl-2)-associated X protein and phosphorylated p53, and decreased the level of Bcl-2. The combination treatment increased microtubule-associated protein 1 light chain 3 and apoptosis-related gene expression. It was also determined that $\mathrm{TMZ}+$ acteoside induced apoptosis and autophagy through the mitogen-activated protein kinase
\end{abstract}

Correspondence to: Professor Jwa-Jin Kim, Department of Medical Science, School of Medicine, Chungnam National University, 266 Munhwa-ro, Jung-gu, Daejeon 35015, Republic of Korea

E-mail: kjj1021@naver.com/kjj4827@gmail.com

Professor Jae Ho Park, Department of Pharmaceutical Science, Jungwon University, 85 Munmu-ro, Geosan, Chungbuk 28024, Republic of Korea

E-mail: parkjh@jwu.ac.kr

Key words: temozolomide, acteoside, glioblastoma, autophagy signaling pathway. These findings suggest that acteoside has beneficial effects on TMZ-based glioblastoma therapy.

\section{Introduction}

Glioblastoma is the most common type of malignant primary brain tumor and the most invasive and devastating primary brain tumor $(1,2)$, with a median survival rate of $\sim 18$ months with aggressive multimodality therapy. Current treatment decisions are limited and include radiation, chemotherapy and surgery in combination with the alkylating agent temozolomide (TMZ) $(3,4)$. Despite the availability of aggressive therapies, patients with glioblastoma generally have a poor prognosis (5). TMZ is the major chemotherapeutic drug used in the clinical treatment of malignant glioblastoma (6-8); as an alkylating agent in the imidazotetrazine series, it is able to penetrate the blood-brain barrier (9-11). During malignant glioblastoma progression and extensive invasion throughout the brain, steadily increasing drug resistance to TMZ decreases its therapeutic efficacy $(12,13)$. Accordingly, novel therapeutic drugs to counter glioblastoma are urgently sought.

Previous studies have reported that glioma cells undergo cell death via autophagy, or type II programmed cell death, in response to TMZ $(14,15)$. Autophagy can be inhibited by 3-methyladenine (3-MA) through the conversion of microtubule-associated protein 1 light chain 3 (LC3)-I to LC3-II, thus reducing glioblastoma cell death $(16,17)$; however, the role of autophagy is dependent on the cellular context. With the exception of a cytotoxic role during TMZ action, the induction of autophagy by cellular stress is a cytoprotective process that eliminates stress-induced cytoplasmic aggregates, organelles and macromolecules in mammalian cells through the lysosomal system. In turn, cells involved in maintaining homeostasis receive energy through these catabolic processes $(18,19)$. The complex roles of autophagy suggest that an autophagy activator may have anticancer effects in combination with TMZ treatment by inducing glioblastoma cell autophagy without 
exerting deleterious effects or providing benefits to normal tissues. Of note, TMZ has exhibited synergistic therapeutic effects in combination with vitamin $\mathrm{D}$, including suppressed cell viability and enhanced autophagy in glioblastoma cells. Furthermore, the combination of TMZ and vitamin D has been shown to exert anticancer effects in vivo, including reduced tumor size and prolonged survival rate. These studies suggest that combination treatment may have synergistic anticancer effects via autophagic mechanisms in TMZ-based glioblastoma therapy (15).

Acteoside is a phenylethanoid glycoside that is widely distributed in several tonified traditional Chinese herbal medicines $(20,21)$. A number of pharmacological actions, including antioxidant, anticancer, anti-inflammatory, antinephritic and antimetastatic actions, have been associated with acteoside (22-24). Previous studies have demonstrated that acteoside has protective effects against carbon tetrachlorideand D-galactosamine-induced liver injury. The mechanisms underlying the protective effects of acteoside are likely related to its capacity to inhibit P450-mediated bioactivation and free radical scavenging effects induced by carbon tetrachloride exposure $(25,26)$.

Therefore, evidence suggests that both acteoside and TMZ induce anticancer effects through cell death. However, their association and anticancer effects in the context of glioblastoma remain to be elucidated. Therefore, the objective of the present study was to verify the synergistic anticancer effects of acteoside combined with TMZ in glioblastoma therapy. A cell viability assay was performed to analyze the anticancer effects of the combination treatment in C6 glioblastoma cells (a rat glioblastoma cell line), and the results were compared with those following treatment with TMZ alone. To further examine the mechanism of cell death caused by cotreatment with acteoside and TMZ, apoptosis- and autophagy-related genes in C6 cells were examined.

\section{Materials and methods}

Cell culture. The C6 rat glioblastoma cell line was purchased from American Type Culture Collection (Rockville, MD, USA). The cells were cultured under sterile conditions at $37^{\circ} \mathrm{C}$ in a humid environment with $5 \%$ of $\mathrm{CO}_{2}$ in Dulbecco's modified Eagle's medium (DMEM) supplemented with $10 \%$ fetal bovine serum (FBS), and 1\% antibiotics and antimycotics (all Welgene, Daegu, Korea).

Plant material. Abeliophyllum distichum Nakai [voucher no. Park1001(ANH)] was collected in Misun-hyang Theme park, Seongbul-Mountain Recreation Forest, 78, Chungmin-rogigok-gil, Goesan-eup, Goesan-gun, Chungcheongbuk-do, Korea.

Callus induction. To induce callus formation (27), $1-\mathrm{cm}^{2}$ leaf explants were isolated from the fresh plants. The explant was cultured on Murachige and Skoog medium (4\% sucrose, $0.9 \%$ agar supplemented with $1 \mathrm{mg} / \mathrm{l}$ naphthalene acetic acid and $1 \mathrm{mg} / 1$ 2,4-dichlorophenoxyacetic acid, $\mathrm{pH} 5.7$ ) at $25^{\circ} \mathrm{C}$. The callus was induced 20 days later. A sufficient quantity of acteoside was obtained through subculture to separate and purify acteoside from the callus (Fig. 1). Different batches of purified acteoside were used in each experiment. Each experiment was performed three times using a different batch.

Isolation and purification. The ethylacetate fractions were concentrated in vacuo and used as a sample for the purification of acteoside. Acteoside was isolated and purified by the Accelerated Chromatographic Isolation system (Isolera $^{\mathrm{TM}}$ Spektra, Biotage, Uppsala, Sweden) using SNAP KPHOSPHO-SIL and SNAP Ultra Cartridges (Biotage). The acteoside purified from the callus was quantitatively analyzed using the standard acteoside by HPLC-PDA analysis. Finally, acteoside of $\geq 95 \%$ purity was obtained from the callus and used as a sample for chemotherapy of temozolomide-based glioblastoma.

3-(4,5-Dimethylthiazol-2-yl)-,5-diphenyltetrazolium bromide (MTT) assay. To identify the TMZ half maximal inhibitory concentration $\left(\mathrm{IC}_{50}\right.$ value) against $\mathrm{C} 6$ cells, $1 \times 10^{4}$ cells in single cell suspensions were seeded into individual wells of 96-well plates and incubated for $24 \mathrm{~h}$ at $37^{\circ} \mathrm{C}$ prior to $\mathrm{TMZ}$ treatment at the indicated concentrations $(1,5,10$, and $20 \mathrm{mM})$ at $37^{\circ} \mathrm{C}$ for $24 \mathrm{~h}$. Following determination of the $\mathrm{TMZ} \mathrm{IC}_{50}$ value as $5 \mathrm{mM}$, the cells were treated for $24 \mathrm{~h}$ with $5 \mathrm{mM} \mathrm{TMZ}, 50 \mu \mathrm{M}$ acteoside, or a combination of the two $(5 \mathrm{mM} \mathrm{TMZ}$ and $50 \mu \mathrm{M}$ acteoside). MTT solution (5 $\mathrm{mg} / \mathrm{ml})$ was added to each well (10 $\mu \mathrm{l}$ ) and cultured at $37^{\circ} \mathrm{C}$ for $2 \mathrm{~h}$. Subsequently, the medium was removed and DMSO was added at a volume of $200 \mu \mathrm{l}$ each and reacted at room temperature for $30 \mathrm{~min}$. The absorbance at $595 \mathrm{~nm}$ was measured to determine cell viability.

Wound-healing assay. A C6 cell suspension in $1 \mathrm{ml}$ was cultured in a 12-well plate and grown to confluence. The cells were treated with TMZ, acteoside or TMZ + acteoside and then scratched with a sterile $10-\mu 1$ pipette tip to create an artificial wound. At 0 and $24 \mathrm{~h}$ post-wounding, digital images of the wound healing process were captured using an inverted microscope. Cell migration was quantified by measuring the size of the scar at 0 and $24 \mathrm{~h}$ using the image analyzing software, ImageJ 1.43u/Java1.6.0_22 software (NIH, Bethesda, Maryland, USA). Each experiment was performed three times and the measurements were performed in triplicate.

Immunoblotting. The C6 cells were treated with TMZ, acteoside or TMZ + acteoside for $24 \mathrm{~h}$. Cells were lysed on ice by the RIPA (25 mM Tris- $\mathrm{HCl}, \mathrm{pH} 7.5,150 \mathrm{mM} \mathrm{NaCl}, 1 \%$ Nonidet P-40, $1 \%$ sodium deoxycholate, $0.1 \%$ SDS) lysis buffer supplemented with protease and phosphatase inhibitors cocktail (Roche, Basel, Switzerland) for $30 \mathrm{~min}$. After centrifugation at $18,341 \mathrm{x}$ g for $15 \mathrm{~min}$ at $4^{\circ} \mathrm{C}$, supernatant was obtained. Protein concentration was determined using Bradford Protein Assays (Bio-rad, Hercules, CA, USA). Equal amounts of total proteins $(30 \mu \mathrm{g})$ were loaded into single wells and fractionated via electrophoresis via a 10-15\% SDS-PAGE. The proteins were electrotransferred to polyvinylidene difluoride membranes (EMD Millipore, Bedford, MA, USA). Following incubation in blocking solution containing 5\% nonfat milk in Tween/Tris-buffer saline for $30 \mathrm{~min}$ at room temperature. The blots were probed with 1:1,000-diluted primary antibodies (cat. no. 9662, caspase 3; Cell Signaling Technology, Inc., Danvers, MA, USA), B0-cell lymphoma 2 (sc-7382, Bcl-2), 
Bcl-2-associated X protein (cat. no. 2772, Bax), phosphorylated (p-)p53 (sc-101762), total-p53 (sc-6243), $\beta$-actin (cat. no. 4970; all; Santa Cruz Biotechnology, Inc., Dallas, TX, USA), LC-3 (L8918, Sigma; Merck KGaA, Darmstadt, Germany), Rab7 (cat. no. 9367, Cell Signaling Technology,Inc.), p62 (cat. no. 114), p-p38 (cat.no. 9211), total-p38 (cat. no. 9212), p-c-Jun N-terminal kinase (cat. no. 9251, p-JNK), total-JNK (cat. no. 9252), p-extracellular signal-regulated kinase (cat. no. 9101, p-ERK), total-ERK (cat. no. 9102; all Cell Signaling Technology, Inc.) overnight at $4^{\circ} \mathrm{C}$. This was followed by incubation with horseradish peroxidase-conjugated secondary antibodies $(1: 2,000$; LF-SA8001A, Goat Anti-Mouse IgG-HRP) or LF-SA8002A (Goat Anti-Rabbit IgG-HRP), AB Frontier, Seoul, Korea.) for $2 \mathrm{~h}$ at room temperature. The proteins were then visualized by exposure to Chemi-Doc (Bio-Rad Laboratories, Inc., Hercules, CA, USA).

Immunofluorescence staining. Lysosomal-associated membrane protein 1 (LAMP1) and LC3 are markers for lysosomal and autophagy. The cells $\left(1 \times 10^{5}\right.$ cells/well $)$ were prepared on sterilized glass coverslips (BD Biosciences, Franklin Lakes, NJ, USA) in triplicate. Following treatment with TMZ, acteoside or $\mathrm{TMZ}+$ acteoside for $24 \mathrm{~h}$, the cells were fixed in $4 \%$ paraformaldehyde for $30 \mathrm{~min}$, blocked with 5\% normal chicken serum (S-3000; Vector Laboratories, Inc., Burlingame, CA, USA), $0.1 \%$ Triton X-100 and then incubated $1 \mathrm{~h}$ for permeabilization and to block non-specific protein-protein interactions. The cells were then incubated with the anti-LAMP1 (1:200; sc-19992, Santa Cruz Biotechnology, Inc.) and anti-LC3 (1:400; PM036, MBL International, Woburn, MA, USA) overnight at $4^{\circ} \mathrm{C}$. The cells were then washed twice with PBS and incubated for an additional $2 \mathrm{~h}$ in the dark with a mixture of Alexa Fluor 488 and 594 secondary antibodies (1:200; Alexa Fluor 488 (A-11008) and 594 (A-11007), Thermo Fisher Scientific, Inc., Waltham, MA, USA) and washed again with PBS. The nuclei were stained using 4,6-diamidino-2-phenylindole (Sigma; Merck KGaA), and then washed twice with PBS. The slides were then mounted and images were captured under an LSM-700 laser confocal microscope.

Flow cytometry. The cells were analyzed for Mitotracker Green and MitoSOX by flow cytometry using a FACSCanto II flow cytometer, as indicated by the manufacturer (BD Biosciences). Following two washes with PBS, the cells were fixed in $4 \%$ paraformaldehyde for $30 \mathrm{~min}$ at room temperature and permeabilized with $0.25 \%$ Triton X-100 in PBS for $20 \mathrm{~min}$. The cells were stained with primary antibodies for overnight at $4^{\circ} \mathrm{C}(1: 200)$ and then with secondary antibodies for $1 \mathrm{~h}$ on ice. Following two washes with PBS, the cells were fixed in $4 \%$ paraformaldehyde and assayed immediately. The flow cytometry data were collected using 10,000 cells and were analyzed using FlowJo 7.6.1 software (Tree Star, Inc., Ashland, OR, USA).

Statistical analysis. All data were analyzed using GraphPad Prism 5.0 and are presented as the mean \pm standard error of the mean. Data were analyzed with one-way analysis of variance with Tukey's multiple-comparisons post hoc test for the comparison of mean values among multiple groups. $\mathrm{P}<0.05$ was considered to indicate a statistically significant difference.

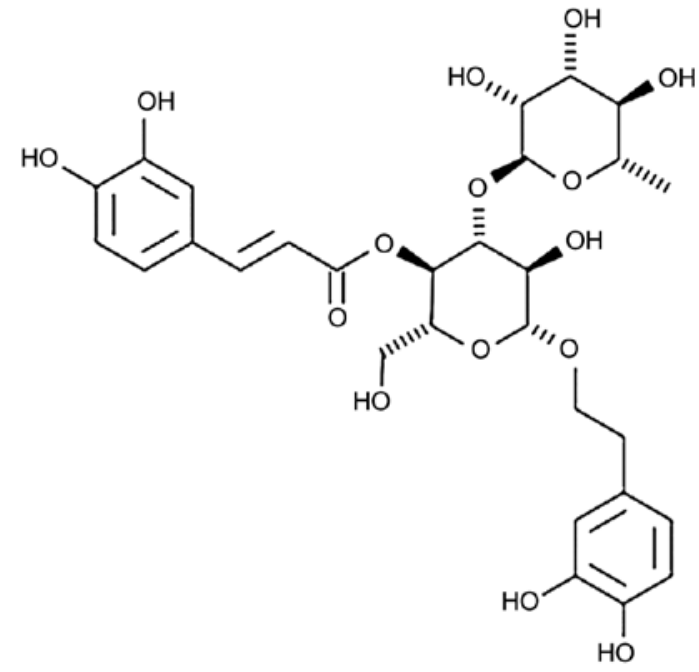

Figure 1. Chemical structure of acteoside.

\section{Results}

Cotreatment with TMZ and acteoside suppresses glioblastoma cell proliferation and migration. Combined treatment with TMZ and acteoside inhibited the viability of C6 cells. TMZ reduced cell viability in a dose-dependent manner (Fig. 2A), whereas acteoside alone had no significant effect at any treatment level (Fig. 2B). Following incubation in culture medium containing TMZ with or without acteoside for $24 \mathrm{~h}$, an MTT assay was performed to compare the cytotoxicities of different treatment levels. TMZ significantly suppressed cell viability, whereas acteoside did not significantly suppress cell growth. Combined treatment with TMZ and acteoside markedly reduced cell viability (Fig. 2C). Treatment with TMZ or acteoside applied alone reduced wound-healing ability (Fig. 3A). The wound distance (\%) was measured using the results shown in Fig. 3A with ImageJ software. The wound distance increased significantly following combination treatment (Fig. 3B), compared with either individual treatment. These results indicate that cotreatment with TMZ and acteoside was an effective inhibitor of glioblastoma cell proliferation and migration.

Acteoside and TMZ affect apoptosis synergistically in C6 cells. The results of the western blot analysis revealed that the levels of cleaved caspase-3, Bax and phosphorylated p53 were higher and the levels of Bcl-2 were lower following combination treatment with $\mathrm{TMZ}$ and acteoside than following treatment with TMZ alone (Fig. 4A). Acteoside-mediated mitochondrial function and the generation of reactive oxygen species (ROS), which are key mediators of apoptotic signaling, were then observed in the TMZ-treated C6 cells. To verify mitochondrial mass, fluorescence-activated cell sorting analysis was performed using MitoTracker Green. Cotreatment with TMZ and acteoside resulted in a higher mitochondrial mass than treatment with TMZ alone (Fig. 4B). ROS generation was significantly higher following cotreatment with TMZ and acteoside than treatment with TMZ alone (Fig. 4C). These results suggest that ROS generation and mitochondrial function are important in apoptosis induced by treatment with $\mathrm{TMZ}+$ acteoside. 

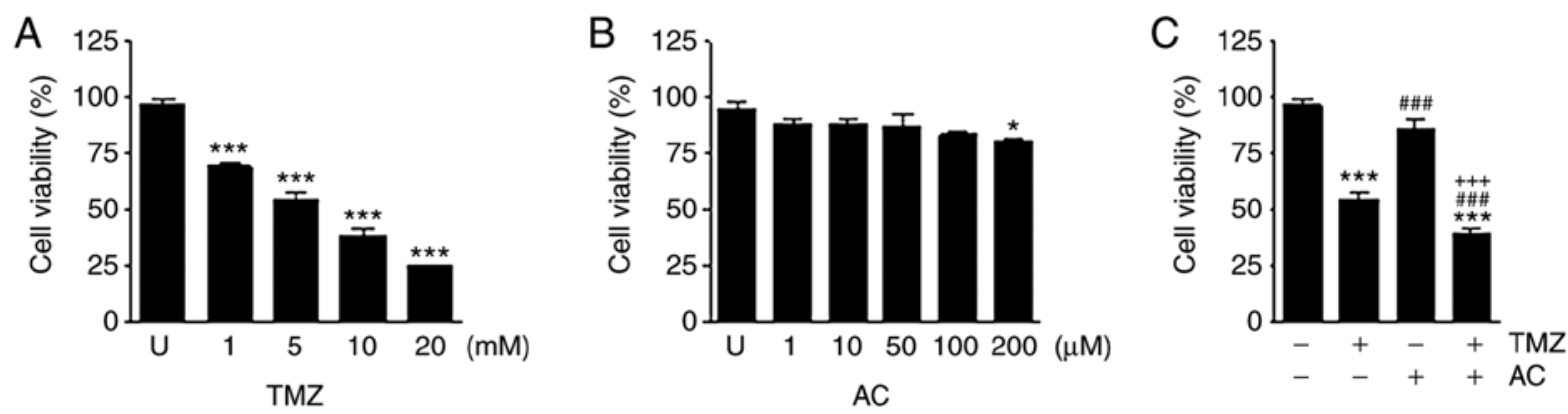

Figure 2. Effects of acteoside on cell viability. (A) C6 cells were treated with various concentrations of TMZ for 24 h and cytotoxicity was assessed by cell viability with 3-(4,5-dimethylthiazol-2-yl)-,5-diphenyltetrazolium bromide. (B) C6 cell were treated with 1, 10, 50, 100 and $200 \mu \mathrm{M}$ acteoside for $24 \mathrm{~h}$. (C) Cell viability of $\mathrm{C} 6$ cells treated with acteoside $(50 \mu \mathrm{M})$ and TMZ $(5 \mathrm{mM}) .{ }^{*} \mathrm{P}<0.05$ and ${ }^{* * *} \mathrm{P}<0.001$ vs. $\mathrm{U}$ group; ${ }^{* \# \#} \mathrm{P}<0.01$ vs. TMZ group; ${ }^{+++} \mathrm{P}<0.001$ vs. AC group. $\mathrm{U}$, untreated; TMZ, temozolomide; AC, acteoside.

A

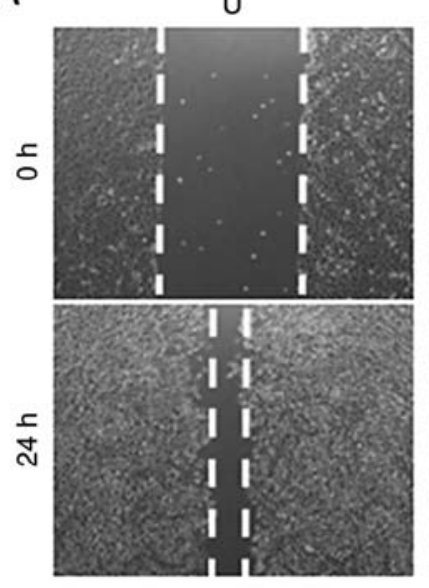

TMZ

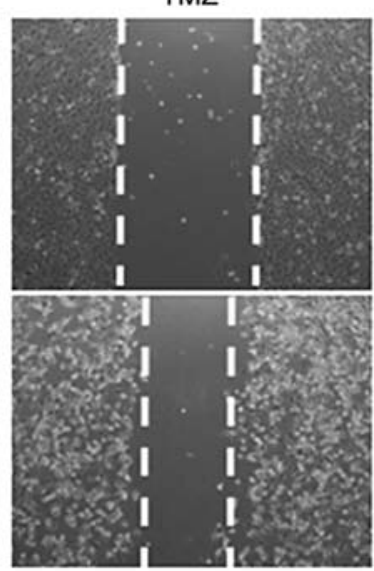

$\mathrm{AC}$

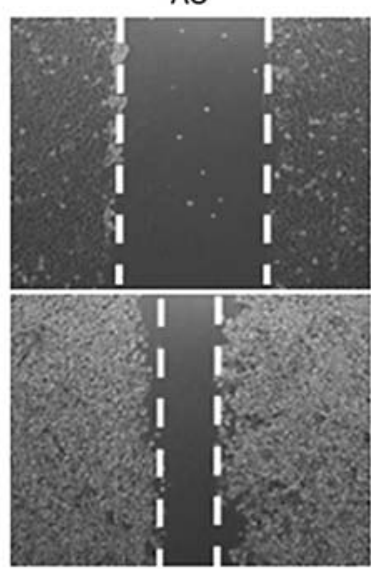

$\mathrm{TMZ}+\mathrm{AC}$

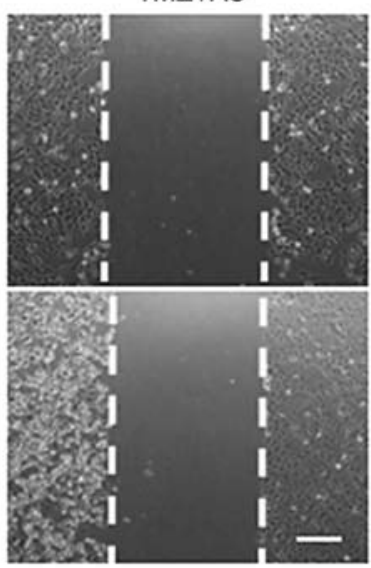

B

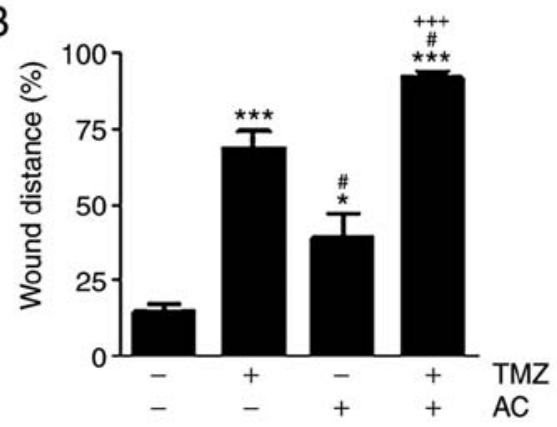

Figure 3. Combination treatment with TMZ and acteoside prevents cell migration in a wound-healing assay. (A) Representative microscopic images (scale bar, $500 \mu \mathrm{m}$ ) of a wound-healing assay using C6 cells treated with $5 \mathrm{mM} \mathrm{TMZ}, 50 \mu \mathrm{M}$ acteoside or TMZ $(5 \mathrm{mM})+$ acteoside ( $50 \mu \mathrm{M})$ for $24 \mathrm{~h}$. (B) Graph showing wound distance; data are presented as the mean + standard error of the mean of at least three independent experiments. ${ }^{*} \mathrm{P}<0.05$ and ${ }^{* * *} \mathrm{P}<0.001 \mathrm{vs}$. $\mathrm{U}$ group; ${ }^{\text {"}} \mathrm{P}<0.05$ vs. TMZ group; ${ }^{+++} \mathrm{P}<0.001$ vs. AC group. $\mathrm{U}$, untreated; TMZ, temozolomide; AC, acteoside.

Cotreatment with TMZ and acteoside induces autophagy in C6 cells. TMZ has been reported to induce autophagy $(28,29)$; therefore, the present study examined the extent to which autophagy was induced by TMZ + acteoside treatment. The C6 cells were treated with TMZ with or without acteoside; after $24 \mathrm{~h}$, and the cells were stained for immunofluorescence to detect LAMP1 and LC3 as markers of autophagy. Higher expression levels of LAMP1 and LC3 were observed following the combination treatment (Fig. 5A). Autophagy-related protein expression was also examined, and it was found that TMZ induced the conversion of LC3-I to LC3-II, which is a signature of autophagosome generation. A higher rate of conversion of LC3-I to LC3-II was observed following cotreatment with TMZ and acteoside than treatment with TMZ alone. The expression levels of Rab7 and p62 indicated autophagy induction in the TMZ-treated cells (Fig. 5B). These findings suggest that cotreatment with TMZ and acteoside enhanced the autophagic process in glioblastoma cells.

Acteoside induces MAPK pathway gene expression in TMZ-based treatment. Previous studies have demonstrated that TMZ regulates the MAPK pathway, including $\mathrm{p} 38$, JNK and ERK (30). Therefore, the present study investigated whether acteoside affects TMZ-related MAPK gene expression. The C6 
A
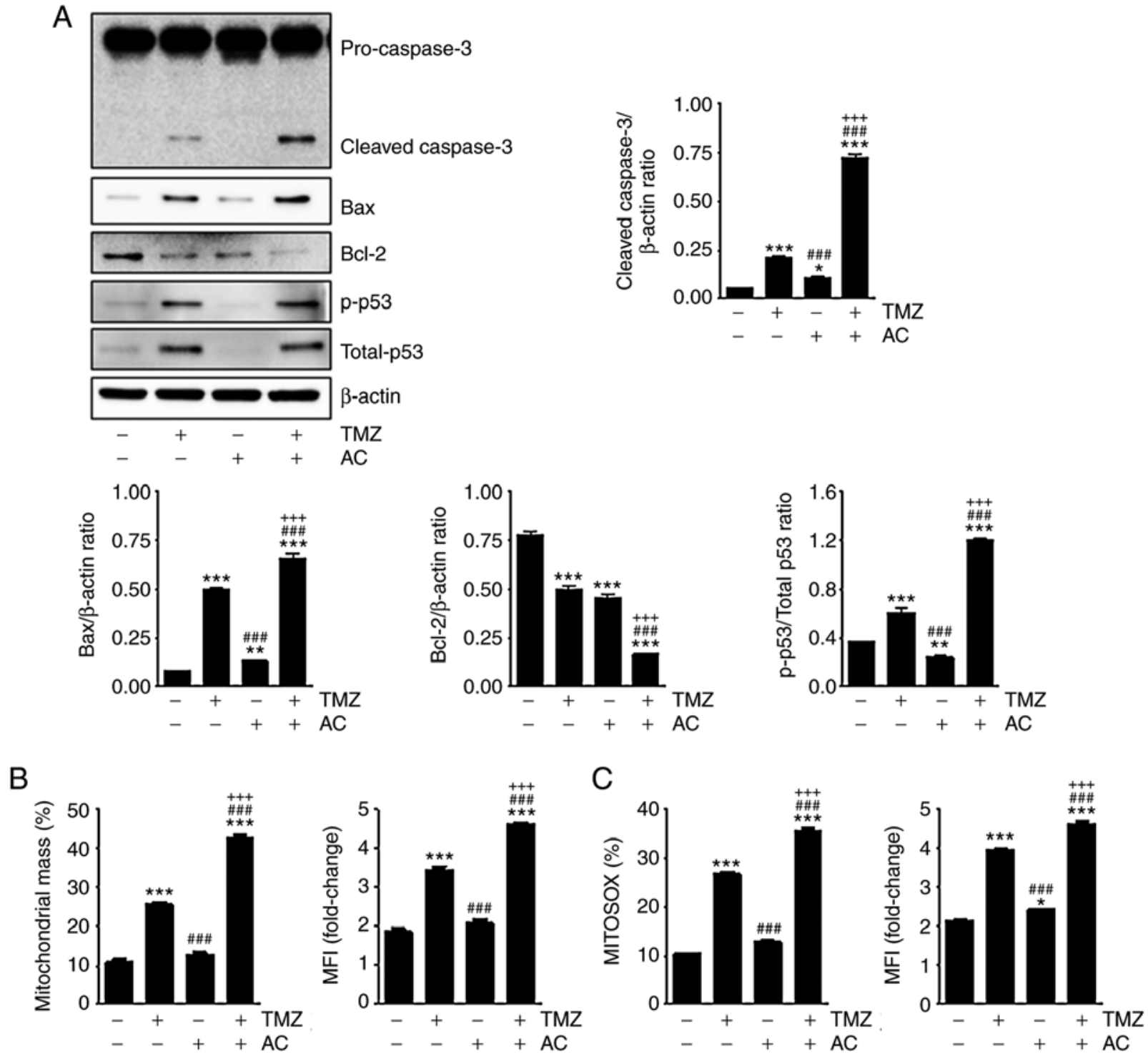

Figure 4. Acteoside and TMZ affect apoptosis synergistically in C6 cells. (A) Western blot analysis revealed the levels of cleaved caspase-3, Bax, Bcl-2, p-p53, total-p53 and $\beta$-actin in C6 cells treated with TMZ (5 mM), acteoside $(50 \mu \mathrm{M})$, and TMZ $(5 \mathrm{mM})+$ acteoside $(50 \mu \mathrm{M})$ for $24 \mathrm{~h}$. Bar graphs indicated the density of cleaved caspase-3, Bax, Bcl2, total-p53, and p-p53. (B) MitoTracker Green flucorescence was measured by FACS analysis for mitochondrial mass. MFI indicates mitochondrial mass. (C) C6 cells were stained for MitoSOX to detect ROS by FACS analysis. Bar graph shows ROS fluorescence intensity. ${ }^{*} \mathrm{P}<0.05,{ }^{* *} \mathrm{P}<0.01$ and ${ }^{* * *} \mathrm{P}<0.001$ vs. untreated group; ${ }^{\# \# "} \mathrm{P}<0.001$ vs. TMZ group; ${ }^{+++} \mathrm{P}<0.001$ vs. AC group. TMZ, temozolomide; AC, acteoside; Bcl-2, B-cell lymphoma 2; Bax, Bcl-2-associated X protein; p-, phosphorylated; FACS, fluorescence-activated cell sorting; MFI, mean fluorescence intensity; ROS, reactive oxygen species.

cells were treated with TMZ with or without acteoside. After $24 \mathrm{~h}, \mathrm{TMZ}$ treatment resulted in higher expression levels of p-p38, p-JNK and p-ERK. The expression of TMZ-regulated genes was significantly higher following TMZ + acteoside treatment than treatment with TMZ alone (Fig. 6). These observations suggest that acteoside affects TMZ-based therapeutic mechanisms via the MAPK pathway.

\section{Discussion}

The results of the present study establish that the combined treatment with TMZ with acteoside offers therapeutic potential for glioblastoma treatment, and provide evidence that chemosensitization to a combination of TMZ and acteoside can occur through autophagy enhancement. As the mutation of glioblastoma cells can lead to apoptotic pathway inactivation, the induction of autophagy by TMZ + acteoside cotreatment may represent an alternative method for glioblastoma therapy. However, whether autophagy is the sole mechanism for glioblastoma therapy with TMZ + acteoside cotreatment remains to be elucidated.

Autophagy is an essential cellular mechanism for the degradation of proteins and cytoplasmic organelles. The catabolic advantage of induced autophagy may be important in stressful conditions; therefore, the induction of autophagy may be an adaptive mechanism for cell death prevention (31-33). Previous studies have reported that reduced autophagy is correlated with cancer (34-36). In addition, several proteins and signaling pathways associated with autophagy are deregulated during malignant transformation, resulting in a reduction in autophagic activity. High expression levels of LC3 have also been associated with increased 
A
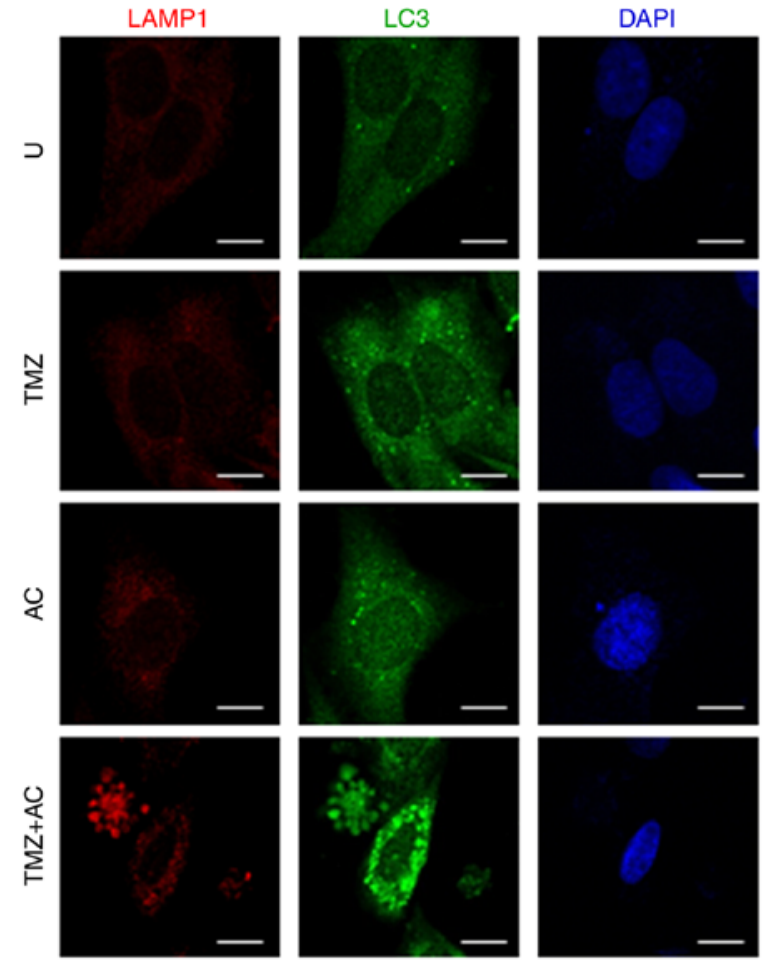
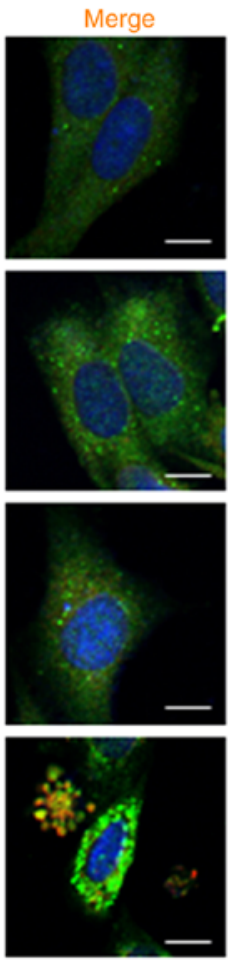

B
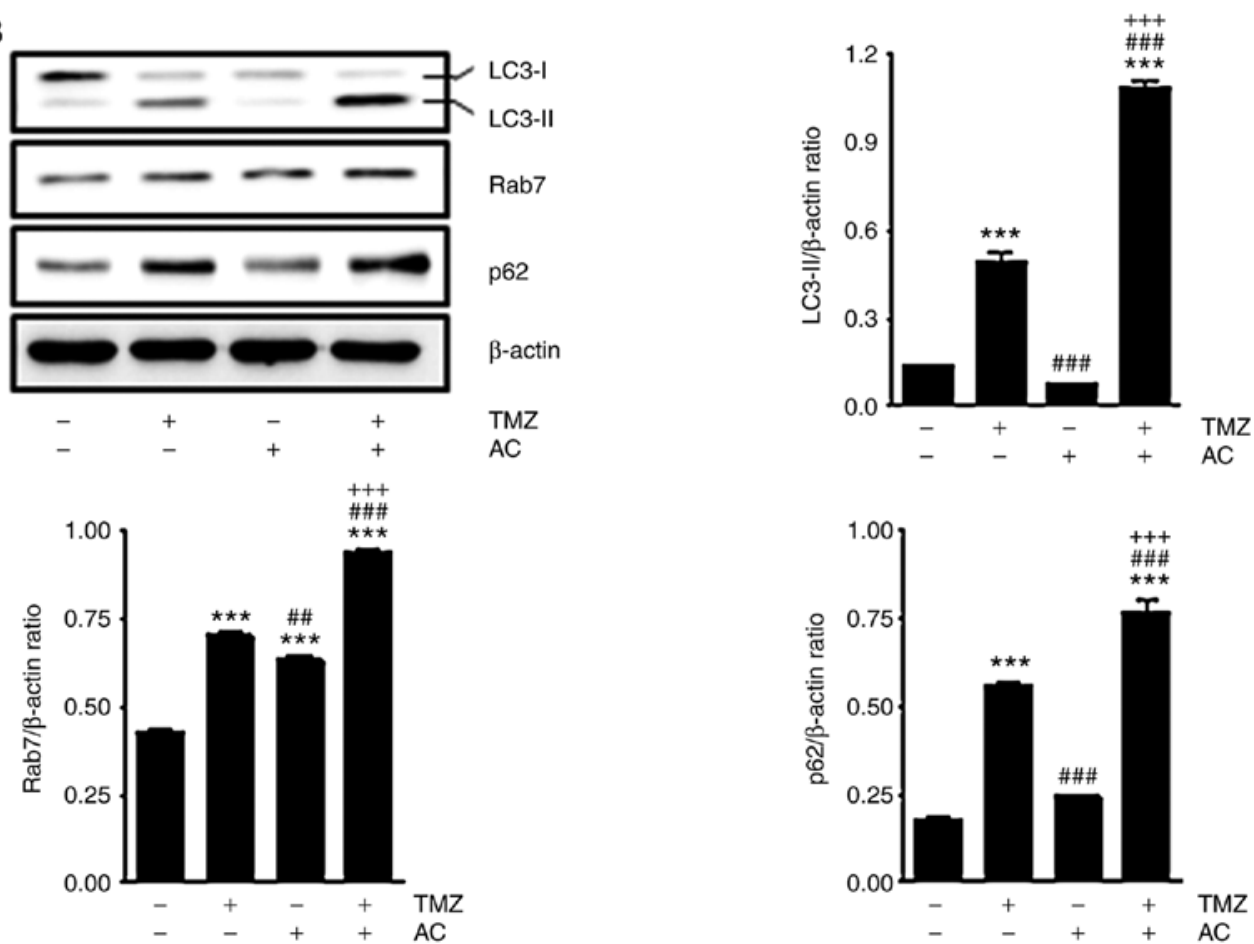

Figure 5. Cotreatement with TMZ + acteoside induces autophagy in C6 cells. (A) Confocal microscopy shows expression of autophagosomes with anti-LAMP1 and anti-LC3 antibodies in C6 cells treated for $24 \mathrm{~h}$ with TMZ (5 mM) + acteoside (50 $\mu$ M). Red, LAMP1; Green, LC3; Blue, DAPI. Scale bar, $10 \mu \mathrm{m}$. (B) Western blot analysis showing the levels of LC3, Rab7, p62 and $\beta$-actin in cells treated with acteoside with or without TMZ for 24 h. Band densities of LC3-II, Rab7 and p63 are indicated as bar graphs. ${ }^{* * *} \mathrm{P}<0.001$ vs. Untreated group; ${ }^{\#} \mathrm{P}<0.01$ and ${ }^{\# \#} \mathrm{P}<0.001$ vs. TMZ group; ${ }^{+++} \mathrm{P}<0.001$ vs. AC group. U, Untreated; TMZ, temozolomide; AC, acteoside. LC3, microtubule-associated protein 1 light chain 3; LAMP1, lysosomal-associated membrane protein 1; 4,6-diamidino-2-phenylindole.

survival rates in patients with glioblastoma with poor performance scores $(37,38)$. Similarly, the results of the present study indicate that the restoration of normal autophagy may be a latent strategy for glioblastoma therapy, and may serve as a mechanism for the restriction of abnormal tumor cell growth.
In normal autophagy, particular cytoplasmic components are isolated within autophagosomes which then fuse with a lysosome to be degraded and recycled $(39,40)$. When autophagy is upregulated, autophagosome formation rates surpass lysosomal degradation rates; this condition is termed autophagic stress. If stress or abnormal autophagy continues, 

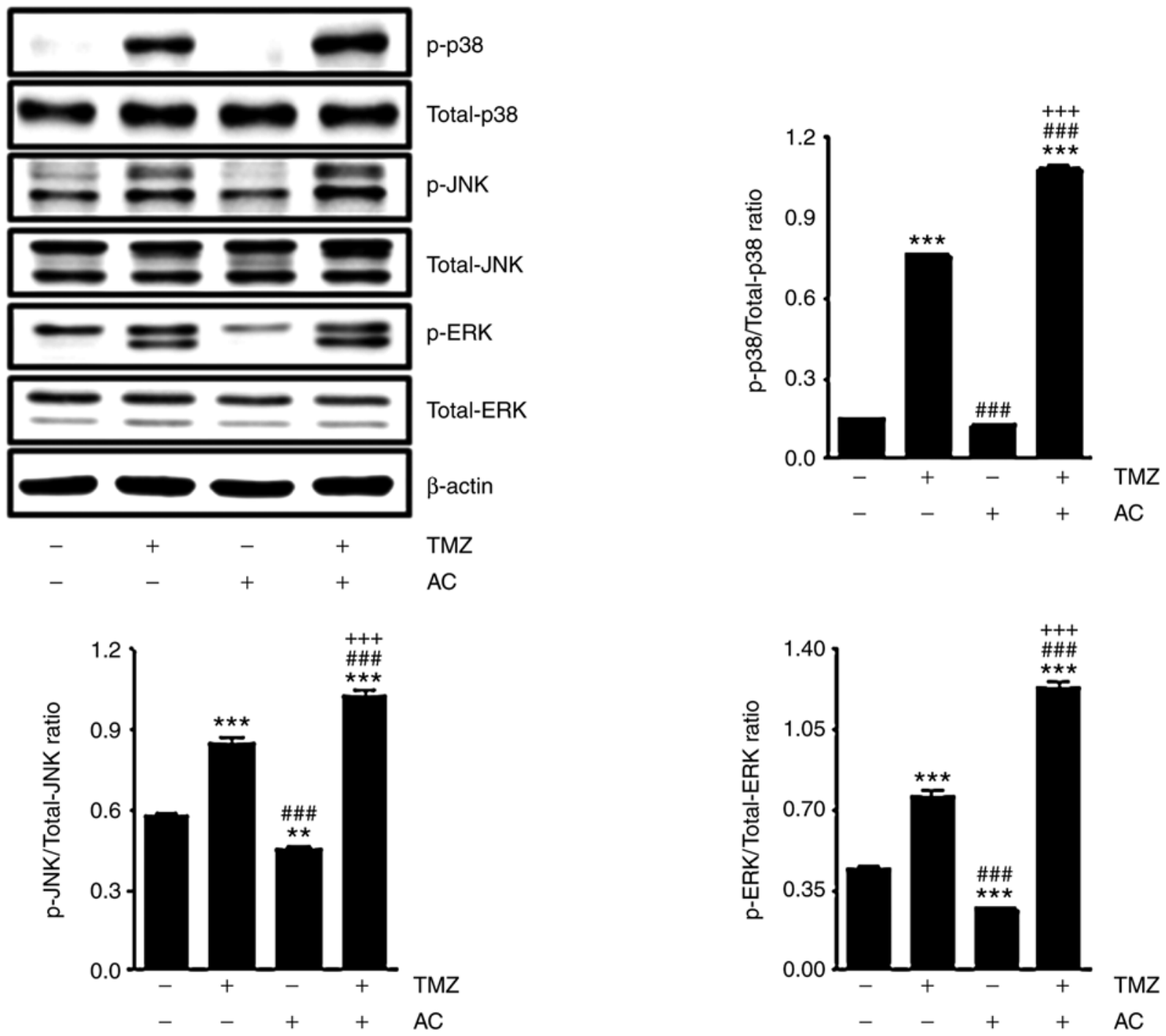

Figure 6. Acteoside induces mitogen-activated protein kinase pathway gene expression in TMZ-based treatment. Western blot analysis showed the levels of p-p38, total-p38, p-JNK, total-JNK, p-ERK and total-ERK in cells treated with TMZ (5 mM) with or without acteoside (50 $\mu \mathrm{M})$ for $24 \mathrm{~h}$. The ratio of p-p38, p-JNK and p-ERK compared with total-p38, total-JNK and total-ERK, respectively, are shown in bar graphs. ${ }^{* *} \mathrm{P}<0.01$ and ${ }^{* * *} \mathrm{P}<0.001$ vs. untreated group;

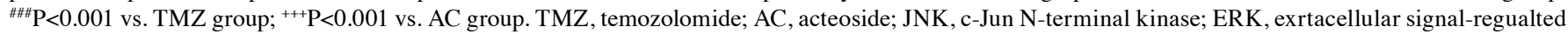
kinase; p-, phosphorylated.

cell death can occur via energy depletion or changes in the beclin-1/Bcl-2 balance. Apoptosis can also be triggered by autophagosome hyperactivity, engulfing cytoplasmic organelles including the mitochondria or endoplasmic reticulum (41). It has been suggested that general autophagy can induce cell death during normal cytoplasmic and organelle turnover in healthy cells. In this process, the cell 'cannibalizes' itself from the inside, a key characteristic of type II programmed cell death. Although the mechanisms by which acteoside enhances the therapeutic effect of TMZ-based glioblastoma therapy remain to be fully elucidated, the anticancer effects exhibited by the combination treatment examined in the present study may be associated with these autophagic mechanisms.

Acteoside is a phenyl-ethanoid glycoside derived from plants $(22,26)$. Previous studies have reported on the various biological activities of acteoside. The changes in the expression levels of cleaved caspase- 3 and LC 3 in the present study demonstrate that treatment with a combination of TMZ and acteoside induced apoptosis and autophagy in C6 cells (Figs. 4 and 5). The combination treatment was shown to have a synergistic effect on glioblastoma cells. Although other possible mechanisms of these synergistic effects remain to be elucidated, the present study identified the induction of autophagy as a crucial tumoricidal mechanism of acteoside chemosensitization during TMZ-based glioblastoma therapy.

\section{Acknowledgements}

Not applicable.

\section{Funding}

This study was supported by the National Research Foundation of Korea (NRF) grants funded by the Korean government (MSIP; grant nos. 2016R1C1B1015811 and 2017R1D1A3B03036420). 


\section{Availability of data and materials}

All data generated or analyzed during this study are included in this published article.

\section{Authors' contributions}

TWH and JJK drafted the manuscript and performed the experiments. TWH, DHK, DBK, TWJ, and GHK performed the experiments and data interpretation. KAY contributed materials/analysis tools and helped in data analysis. MM, DEC, JHP, and JJK designed the experiments, provided critical suggestions for the manuscript, and reviewed and revised the manuscript. All authors read and approved the final manuscript.

\section{Ethics approval and consent to participate}

Not applicable.

\section{Patient consent for publication}

Not applicable.

\section{Competing interests}

The authors declare that they have no competing interests.

\section{References}

1. Friedman HS, Kerby $\mathrm{T}$ and Calvert H: Temozolomide and treatment of malignant glioma. Clin Cancer Res 6: 2585-2597, 2000.

2. Mrugala MM and Chamberlain MC: Mechanisms of disease: Temozolomide and glioblastoma-look to the future. Nat Clin Pract Oncol 5: 476-486, 2008.

3. Agarwala SS and Kirkwood JM: Temozolomide, a novel alkylating agent with activity in the central nervous system, may improve the treatment of advanced metastatic melanoma. Oncologist 5: 144-151, 2000.

4. Stevens MF, Hickman JA, Stone R, Gibson NW, Baig GU, Lunt E and Newton CG: Antitumor imidazotetrazines. 1. Synthesis and chemistry of 8-carbamoyl-3-(2-chloroethyl)imidazo[5,1-d]-1,2,3, 5-tetrazin-4(3 H)-one, a novel broad-spectrum antitumor agent. J Med Chem 27: 196-201, 1984.

5. Fulda S: Cell death-based treatment of glioblastoma. Cell Death Dis 9: 121, 2018.

6. Chen PH, Shen WL, Shih CM, Ho KH, Cheng $\mathrm{CH}$, Lin CW, Lee CC, Liu AJ and Chen KC: The CHAC1-inhibited Notch3 pathway is involved in temozolomide-induced glioma cytotoxicity. Neuropharmacology 116: 300-314, 2017.

7. Oshiro S, Tsugu H, Komatsu F, Ohmura T, Ohta M, Sakamoto S, Fukushima T and Inoue T: Efficacy of temozolomide treatment in patients with high-grade glioma. Anticancer Res 29: 911-917, 2009.

8. van den Bent MJ: Adjuvant treatment of high grade gliomas. Ann Oncol 17 (Suppl 10): x186-x190, 2006.

9. Shen W, Hu JA and Zheng JS: Mechanism of temozolomide-induced antitumour effects on glioma cells. J Int Med Res 42: 164-172, 2014.

10. Barciszewska AM, Gurda D, Głodowicz P, Nowak S and Naskręt-Barciszewska MZ: A new epigenetic mechanism of temozolomide action in glioma cells. PLoS One 10: e0136669, 2015.

11. Cai X and Sughrue ME: Glioblastoma: New therapeutic strategies to address cellular and genomic complexity. Oncotarget 9: 9540-9554, 2018.

12. Zhang J, Stevens MF and Bradshaw TD: Temozolomide: Mechanisms of action, repair and resistance. Curr Mol Pharmacol 5: 102-114, 2012.
13. Johannessen TC and Bjerkvig R: Molecular mechanisms of temozolomide resistance in glioblastoma multiforme. Expert Rev Anticancer Ther 12: 635-642, 2012.

14. Yan Y, Xu Z, Dai S, Qian L, Sun L and Gong Z: Targeting autophagy to sensitive glioma to temozolomide treatment. J Exp Clin Cancer Res 35: 23, 2016.

15. Bak DH, Kang SH, Choi DR, Gil MN, Yu KS, Jeong JH, Lee NS, Lee JH, Jeong YG, Kim DK, et al: Autophagy enhancement contributes to the synergistic effect of vitamin D in temozolomide-based glioblastoma chemotherapy. Exp Ther Med 11: 2153-2162, 2016.

16. Li C, Liu Y, Liu H, Zhang W, Shen C, Cho K, Chen X, Peng F, Bi Y, Hou X, et al: Impact of autophagy inhibition at different stages on cytotoxic effect of autophagy inducer in glioblastoma cells. Cell Physiol Biochem 35: 1303-1316, 2015.

17. Ni H, Gong Y, Yan JZ and Zhang LL: Autophagy inhibitor 3-methyladenine regulates the expression of LC3, Beclin-1 and ZnTs in rat cerebral cortex following recurrent neonatal seizures. World J Emerg Med 1: 216-223, 2010.

18. Xie Z and Klionsky DJ: Autophagosome formation: Core machinery and adaptations. Nat Cell Biol 9: 1102-1109, 2007.

19. Gutierrez MG, Master SS, Singh SB, Taylor GA, Colombo MI and Deretic V: Autophagy is a defense mechanism inhibiting BCG and Mycobacterium tuberculosis survival in infected macrophages. Cell 119: 753-766, 2004.

20. Wu YT, Wu MT, Lin CC, Chien CF and Tsai TH: Pharmacokinetic studies of chinese medicinal herbs using an automated blood sampling system and liquid chromatography-mass spectrometry. J Tradit Complement Med 2: 33-40, 2012.

21. Inoue M, Sakuma Z, Ogihara Y and Saracoglu I: Induction of apoptotic cell death in HL-60 cells by acteoside, a phenylpropanoid glycoside. Biol Pharm Bull 21: 81-83, 1998.

22. Ohno T, Inoue M, Ogihara Y and Saracoglu I: Antimetastatic activity of acteoside, a phenylethanoid glycoside. Biol Pharm Bull 25: 666-668, 2002.

23. Lee KJ, Woo ER, Choi CY, Shin DW, Lee DG, You HJ and Jeong HG: Protective effect of acteoside on carbon tetrachloride-induced hepatotoxicity. Life Sci 74: 1051-1064, 2004.

24. Peerzada KJ, Faridi AH, Sharma L, Bhardwaj SC, Satti NK, Shashi B and Tasduq SA: Acteoside-mediates chemoprevention of experimental liver carcinogenesis through STAT-3 regulated oxidative stress and apoptosis. Environ Toxicol 31: 782-798, 2016.

25. Zhao J, Liu T, Ma L, Yan M, Zhao Y, Gu Z and Huang Y: Protective effect of acteoside on immunological liver injury induced by Bacillus Calmette-Guerin plus lipopolysaccharide. Planta Med 75: 1463-1469, 2009.

26. Xiong Q, Hase K, Tezuka Y, Tani T, Namba T and Kadota S: Hepatoprotective activity of phenylethanoids from Cistanche deserticola. Planta Med 64: 120-125, 1998.

27. Koh DS, Seo BS and Lee CH: Studies on the in vitro induction of callus from anther culture of Abeliophyllum distichum. J Chonbuk Natl Univ 31: 153-159, 1989 (In Korean).

28. Würstle S, Schneider F, Ringel F, Gempt J, Lämmer F, Delbridge $\mathrm{C}, \mathrm{Wu} \mathrm{W}$ and Schlegel J: Temozolomide induces autophagy in primary and established glioblastoma cells in an EGFR independent manner. Oncol Lett 14: 322-328, 2017.

29. Koukourakis MI, Mitrakas AG and Giatromanolaki A: Therapeutic interactions of autophagy with radiation and temozolomide in glioblastoma: Evidence and issues to resolve. $\mathrm{Br}$ J Cancer 114: 485-496, 2016.

30. Chen Y, Gao F, Jiang R, Liu H, Hou J, Yi Y, Kang L, Liu X, Li Y and Yang M: Down-regulation of AQP4 expression via p38 MAPK signaling in temozolomide-induced glioma cells growth inhibition and invasion impairment. J Cell Biochem 118: 4905-4913, 2017.

31. He C and Klionsky DJ: Regulation mechanisms and signaling pathways of autophagy. Annu Rev Genet 43: 67-93, 2009.

32. Bento CF, Renna M, Ghislat G, Puri C, Ashkenazi A, Vicinanza M, Menzies FM and Rubinsztein DC: Mammalian autophagy: How does it work? Annu Rev Biochem 85: 685-713, 2016.

33. Levine B, Mizushima N and Virgin HW: Autophagy in immunity and inflammation. Nature 469: 323-335, 2011.

34. Fulda S: Autophagy in Cancer Therapy. Front Oncol 7: 128, 2017.

35. Levy JMM, Towers CG and Thorburn A: Targeting autophagy in cancer. Nat Rev Cancer 17: 528-542, 2017.

36. White E: The role for autophagy in cancer. J Clin Invest 125: 42-46, 2015. 
37. Aoki H, Kondo Y, Aldape K, Yamamoto A, Iwado E, Yokoyama T, Hollingsworth EF, Kobayashi R, Hess K, Shinojima N, et al: Monitoring autophagy in glioblastoma with antibody against isoform B of human microtubule-associated protein 1 light chain 3. Autophagy 4: 467-475, 2008.

38. Cj P, Hv E, Vijayakurup V, R Menon G, Nair S and Gopala S High LC3/beclin expression correlates with poor survival in glioma: A definitive role for autophagy as evidenced by in vitro autophagic flux. Pathol Oncol Res, Oct 112017. Doi: $10.1007 / \mathrm{s} 12253-017-0310-7$.
39. Monastyrska I and Klionsky DJ: Autophagy in organelle homeostasis: Peroxisome turnover. Mol Aspects Med 27: 483-494, 2006.

40. Farre JC and Subramani S: Peroxisome turnover by micropexophagy: An autophagy-related process. Trends Cell Biol 14: 515-523, 2004.

41. Singh R and Cuervo AM: Autophagy in the cellular energetic balance. Cell Metab 13: 495-504, 2011. 